Thaís Pires Miranda

\title{
Biodiversidade e áreas de endemismo de hidroides bentônicos (Cnidaria, Hydrozoa) da costa austral da América do Sul e Antártica
}

Biodiversity and areas of endemism of benthic hydroids (Cnidaria, Hydrozoa) from southern South America and Antarctica

São Paulo

2014 


\section{Thaís Pires Miranda}

\section{Biodiversidade e áreas de endemismo de hidroides bentônicos (Cnidaria, Hydrozoa) da costa austral da América do Sul e Antártica}

Biodiversity and areas of endemism of benthic hydroids (Cnidaria, Hydrozoa) from southern South America and Antarctica

Tese apresentada no Instituto de Biociências da Universidade de São Paulo, para o obtenção do Título de Doutor em Ciências Biológicas na Área de Zoologia Orientador: Prof. Dr. Antonio Carlos Maques

São Paulo

2014 
Miranda, Thaís Pires

Biodiversidade e áreas de endemismo de hidroides bentônicos (Cnidaria, Hydrozoa) da costa austral da América do Sul e Antártica

112 páginas

Tese (Doutorado) - Instituto de Biociências da Universidade de São Paulo. Departamento de Zoologia.

1. Biogeografia marinha 2. Diversidade 3.

Endemismo 4. Hydrozoa I. Universidade de São Paulo. Instituto de Biociências. Departamento de Zoologia.

\section{Comissão Julgadora:}




\section{Resumo}

A costa austral da América do Sul (CAAS) e o oceano Austral (OA) possuem grande variedade de habitats e estão historicamente conectados desde a abertura da passagem de Drake. A fauna marinha de ambas as regiões é altamente diversa e muitas espécies possuem distribuições geográficas contraditórias, como é o caso dos hidroides bentônicos (Cnidaria, Hydrozoa). Toda essa heterogeneidade faunística atrai estudos adicionais em biogeografia, envolvendo a busca por áreas de endemismo e outros padrões de distribuição geográfica. Uma atualização do status taxonômico das espécies de hidroides bentônicos da CAAS e OA foi feito, reunindo informações sobre sua riqueza, grau de endemismo, substrato biológico e distribuição geográfica. Um total de 5.621 amostras e 359 morfoespécies de hidroides bentônicos foram levantadas para a área de estudo e checadas taxonomicamente, sendo 256 identificadas até o nível específico. Os registros de presença e ausência ao longo da área foram usados em uma análise de similaridade pelo índice de Bray-Curtis, sendo os resultados sintetizados em "clusters" hierárquicos e nMDS. A taxa de endemismo para ambos a CAAS e OA foi de 54\%, sendo o OA responsável por $88 \%$ dos registros de espécies endêmicas. As espécies de leptotecados foram as que apresentaram maior amplitude de distribuição horizontal e vertical. A maior parte das espécies de hidroides bentônicos levantadas são substrato-generalistas. Treze assembleias ecológicas de hidroides bentônicos foram encontradas ao longo da CAAS e OA, as quais foram relacionadas com a dinâmica oceanográfica da área e comparadas com ecorregiões, realms, assembleias e áreas de endemismo previamente delimitadas para a região. As áreas de endemismo para a CAAS e OA foram hipotetizadas por meio da Análise de Endemicidade (AE) em uma otimização pelo software NDM-VNDM, com uma grade de $10^{\circ}$ latitude $\mathrm{X} 10^{\circ}$ longitude. Nove $(F=0.5)$ e $10(F=1.0)$ áreas consensuais de endemismo foram historicamente relacionadas com o isolamento da Antártica e comparadas com hipóteses já existentes para a CAAS e OA. Todas as áreas foram discutidas considerando-se os processos de vicariância e dispersão, e com relação ao real grau de endemicidade que elas representam. Uma PAE também foi realizada mas apenas com 61 espécies de hidroides bentônicos endêmicos da região acima de $45^{\circ} \mathrm{S}$. Essa análise foi feita com o objetivo de reanalisar os dados previamente publicados por Marques \& Peña Cantero (2010), inserindo um conjunto de dados complementar para a obtenção de uma melhor definição e acurácia nas áreas de endemismo delimitadas para a região. Oito áreas de endemismo foram definidas como subregiões dos padrões previamente encontrados. $\mathrm{O}$ uso de diferentes técnicas e conjuntos de dados biogeográficos são meios alternativos para clarear padrões gerais de áreas de endemismo e também outros padrões relacionados à comunidades ecológicas e estudos em conservação da biodiversidade. 


\section{Abstract}

The southern South America (SSA) coast and the Southern Ocean (SO) have a high differentiation of habitats and are historically connected since the opening of the Drake Passage. The marine fauna of both regions are connected, highly diverse and many species are contradictory distributed, such as the benthic hydroids (Cnidaria, Hydrozoa). All this faunistic heterogeneity calls attention for additional biogeographic investigations, involving the search for areas of endemism and other geographic distribution patterns. An update of the taxonomic status of the species of benthic hydroids from SSA and SO was carried out, gathering information about their richness, endemicity level, biological substrates and geographic distribution. A total of 5.621 samples and 359 morphospecies of benthic hydroids were surveyed for the studied area and taxonomically checked, being 256 identified up to the specific level. The records of presence-absence along the SSA and SO were used in a Bray-Curtis similarity analysis and the results were synthesized in hierarchical clusters and nMDS. The level of endemism at both the SSA and the SO is $54 \%$, being the $\mathrm{SO}$ responsible for $88 \%$ of the records of endemic species. Leptothecate species had the widest horizontal and vertical distribution and most part of the benthic hydroids surveyed were considered substrate-generalists. Thirteen ecological assemblages of benthic hydroids were found along the SSA and SO and compared with the oceanographic dynamics of the area, as well as with earlier ecoregions, realms, assemblages, and areas of endemism delimited for the region. Areas of endemism were hypothesized for the SSA and SO using the Endemicity Analysis (EA) through an optimization performed by the software NDM-VNDM, with a $10^{\circ}$ latitude $\mathrm{X} 10^{\circ}$ longitude grid. Nine $(\mathrm{F}=0.5)$ and $10(\mathrm{~F}=1.0)$ consensus areas of endemism were historically related with the isolation of Antarctica, and compared with previous hypotheses for the SSA and SO. All areas were discussed in the context of vicariance/dispersal processes, and concerning the real degree of endemicity that they represent. PAE was also performed but only with 61 endemic species of benthic hydroids for the region above the $45^{\circ} \mathrm{S}$. This analysis was carried out with the aim to reanalyze previous data already published by Marques \& Peña Cantero (2010), inserting a complementary dataset in order to obtain a better definition and accuracy in the areas of endemism delimited for the region. Eight areas of endemism were defined as subregions of the patterns previously found. The use of different techniques and biogeographic datasets are alternative strategies to clarify general patterns of areas of endemism, as well as other patterns related with ecological communities and studies in conservation biology. 


\section{Capítulo1. Introdução Geral}

\section{BiodiVERSIDADE MARINHA E ENDEMISMO NA COSTA AUSTRAL DA AMÉRICA DO SUL E ANTÁRTICA}

Regiões polares são tradicionalmente consideradas como áreas de baixa diversidade marinha, em especial por serem compreendidas como ambientes de fatores abióticos extremos (Clarke, 2008). No Oceano Austral (OA), por exemplo, o padrão classicamente aceito é que a biodiversidade marinha aumenta em latitudes menores como, por exemplo, nas ilhas subantárticas - entretanto, há poucos estudos empíricos que contrastem a variação latitudinal com a riqueza de espécies para esta região (Clarke \& Johnston, 2003). Em uma outra perspectiva sobre a questão, Clarke \& Johnston (2003) revisaram listas com a diversidade marinha antártica e consideraram o OA como detentor de um alto grau de diversidade e de endemismo em sua fauna.

Estudos com inferências em áreas endemismo para a fauna do OA são escassos (viz., revisão em Marques \& Peña Cantero, 2010 e Miranda et al., 2013), mesmo com o aumento dos estudos sobre a biodiversidade antártica nas últimas décadas (Arntz, 1997; López-González \& Gili, 2000). Como causa/consequência desta escassez, há uma defasagem metodológica, numérica e de extensão geográfica dos estudos em biogeografia marinha, em geral na comparação com o equivalente para o ambiente terrestre. A escassez de estudos em biogeografia marinha pode estar relacionada, talvez, a uma compreensão implícita de uma aparente inexistência de barreiras geográficas no ambiente, ou na dificuldade em caracterizá-las, inclusive sob uma perspectiva histórica (Miranda \& Marques, 2011).

Estudos biogeográficos esbarram em dificuldades taxonômicas como, por exemplo, a confiabilidade das identificações das espécies e, consequentemente, a determinação dos pontos geográficos em que há a real presença de um táxon. Outra dificuldade é a existência de cladogramas no nível específico baseados em dados confiáveis da identificação das espécies, que permitam a inferência das distribuições em um contexto filogenético. Desta forma, o aprofundamento de estudos taxonômicos é fundamental para inferências de áreas de endemismo da biota como um todo ou de comunidades específicas (Miranda \& Marques, 2011). Estas áreas de endemismo constituem a fase inicial dos estudos biogeográficos (Crisci et al., 2000), podendo ser consideradas hipóteses de homologia biogeográfica primária (Morrone, 2001) - por consequência, sua confiabilidade é um passo necessário para inferências posteriores, tais como estudos filogeográficos, ecológicos e evolutivos.

O alto grau de endemicidade da fauna do OA sugere a ocorrência de uma série de processos de especiação em um longo período de isolamento do continente antártico (Clarke \& Crame, 1992; López-González \& Gili, 2000; Clarke \& Johnston, 2003; Clarke et al., 2005; Marques \& Peña Cantero, 2010). Embora majoritariamente isolada, há evidências que sugerem uma afinidade da fauna da Península Antártica com a fauna subantártica da América do Sul (Clarke \& Johnston, 2003; Clarke et al., 2005; Marques \& Peña Cantero, 2010), particularmente devido à presença do Arco Scotia, o qual apresenta uma feição que eventualmente permitiria a comunicação entre as faunas americana e antártica (Clarke \& Johnston, 2003).

Alguns estudos recentes sobre a fauna antártica enfocam comparações entre esta fauna e a subantártica, levando em conta diversos táxons marinhos, tais como briozoários, crustáceos, equinodermos, hidroides, moluscos, poliquetos, poríferos e sipunculídeos (viz., Brandt et al., 1999; De Broyer \& Rauschert, 1999; Cañete et al., 1999; Gerdes \& Montiel, 1999; MühlenhardtSiegel, 1999; Peña Cantero \& García Carrascosa, 1999; Saiz-Salinas \& Pagola-Carte, 1999). 
Entretanto, a fauna marinha subantártica, particularmente a da região magalhânica, é negligenciada em comparação com o conhecimento acumulado para o OA (Brandt et al., 1999; De Broyer \& Rauschert, 1999; Montiel et al., 2005), principalmente em relação aos hidroides bentônicos.

O sudoeste do Atlântico (SOA) e sudeste do Pacífico (SEP) não ficam longe deste quadro de desconhecimento da fauna de hidroides bentônicos, inclusive para regiões mais profundas (viz., Migotto \& Marques, 2006; Galea, 2006a, 2007; Genzano et al., 2009). Estudos faunísticos sobre hidroides bentônicos têm sido feitos há pelo menos sete décadas para a costa da Argentina-Uruguai (Blanco, 1976, 1994; Genzano \& Zamponi, 1997, 2003; Genzano et al., 2009; Rodriguez et al., 2012), Brasil (Vannucci Mendes, 1946; Vannucci, 1949, 1951; Migotto, 1996; Grohmann et al., 2003, 2011; Marques et al., 2003, 2013; Cunha \& Jacobucci, 2010; Miranda et al., 2011) e, mais recentemente para o Chile (Galea, 2006a, 2006b, 2007; Galea et al., 2007a, 2007b, 2007c, 2009a, 2009b; Galea \& Schories, 2012a; Galea et al., 2014). Entretanto, Galea (2006a, 2007) por exemplo, enfatiza que a fauna chilena é negligenciada e pouco documentada em relação ao que se conhece mundialmente, concentrando-se os registros principalmente em trabalhos mais antigos, tais como Jäderholm (1904), Hartlaub (1905) e Leloup (1974). Dados da literatura compilados até o final de 2013 para o subcontinente sul-americano revelam o registro de 615 espécies nominais de hidroides, sendo que destas, 435 foram registradas para a costa austral da América do Sul (CAAS, i.e., região acima dos $22^{\circ} \mathrm{S}$; viz., Oliveira et al., in press). Estes números correspondem a aproximadamente $20 \%$ e 14\%, respectivamente, do total de espécies de hidroides (pólipos e medusas) descritos mundialmente (Schuchert, 1998; Bouillon et al., 2006; Cartwright \& Collins, 2007), o que corrobora o fato do conhecimento taxonômico destas espécies para o SOA e SEP estar longe do ideal.

'Hidroide bentônico' é o termo coloquial que se refere à fase séssil dos organismos das subclasses "Anthoathecata" (não-monofilética) e Leptothecata, ambas inseridas na classe Hydrozoa (filo Cnidaria). Estes organismos têm grande importância no bentos marinho devido ao seu crescimento rápido por reprodução assexuada, seu pioneirismo na colonização de substratos naturais e artificiais, sua enorme diversidade de estratégias reprodutivas sexuadas, além de sua associação com diversos organismos marinhos ( $c f$. ., Gili \& Hughes, 1995; Rosso \& Marques, 1997; Genzano, 1998, 2001, 2002; Genzano \& Rodriguez, 1998; Migotto et al., 2001; Genzano \& San Martín, 2002; Genzano et al., 2003; Oliveira, 2003; Oliveira et al., 2006; Oliveira \& Marques, 2007; Shimabukuro, 2007).

A biodiversidade de hidroides bentônicos não é pouco conhecida somente para a CAAS (i.e., SOA e SEP). Amplas áreas da região antártica também apresentam desconhecimento da fauna de hidroides bentônicos, tornando-se necessário aumentar os conhecimentos faunísticos para ambas as áreas. Dados da literatura desta fauna compilados até 2004 para a Antártica revelam um total de 153 espécies registradas, a maior parte delas apresentando ampla distribuição batimétrica, com ocorrência em regiões de maior profundidade (Peña Cantero, 2004). Em termos taxonômicos, a dominância relativa entre antoatecados e leptotecados ocorre de maneira inversa, ou seja, as populações/espécies de antoatecados concentram-se mais em regiões rasas, enquanto que as de leptotecados concentram-se mais em regiões profundas (Peña Cantero, 2004). Em levantamentos faunísticos extensos é frequente o registro de uma menor ocorrência de antoatecados em relação à leptotecados (Millard, 1975; Schuchert, 2000, 2001; Miranda, 2009). Peña Cantero \& García Carrascosa (1999), por exemplo, ressaltam que, para as regiões magalhânica e antártica, os antoatecados têm sua fauna pouco conhecida, independentemente do esforço e uniformidade amostral.

Algumas semelhanças entre as faunas da Península Antártica e da região magalhânica vêm sendo documentadas (De Broyer \& Rauschert, 1999; Pagès \& Orejas, 1999; Peña Cantero \& 
García Carrascosa, 1999; Saiz-Salinas \& Pagola-Carte, 1999) e associadas à separação tardia entre os continentes antártico e americano (Gerdes \& Montiel, 1999; Lawver \& Gahagan, 2003). Em geral, a interpretação da distribuição desta fauna tem um viés dispersionista, com discussões sobre a localidade de origem e sentido de migração destas faunas, i.e., origem magalhânica e dispersão para a Antártica ou vice-versa ( $c f$. ., Peña Cantero \& García Carrascosa, 1999).

Estudos envolvendo a origem e evolução de organismos marinhos para o SOA, SEP e OA (e.g., Clayton, 1994; Brandt, 1999; Clarke et al., 2004) não possuem, em geral, um enfoque biogeográfico que inclua o uso de diferentes metodologias e análises estritas. Recentemente, Marques \& Peña Cantero (2010) propuseram quatro áreas de endemismo para o OA, usando PAE e uma base de dados de distribuição geográfica do gênero Oswaldella (Leptothecata, Kirchenpaueriidae). Já Miranda et al. (2013) reanalisaram os dados de Oswaldella juntamente com dados adicionais dos gêneros Antarctoscyphus, Mixoscyphus e Staurotheca (Leptothecata, Sertulariidae), e propuseram a existência de oito áreas de endemismo para o OA. Entretanto, estudos focados no uso e comparação de diferentes metodologias para busca de áreas de endemismo ainda são preliminares e raros em biogeografia marinha, talvez devido à necessidade da existência de uma grande quantidade de dados geográficos disponíveis para sua realização. Desta forma, é necessário um aumento no número de estudos taxonômicos que visem comparar e entender o relacionamento entre as faunas antártica e subantártica, sob um contexto biogeográfico e evolutivo, tomando como modelo os hidroides bentônicos.

\section{OBJETIVOS GERAIS}

O estudo teve por principais objetivos:

1) Atualizar os status taxonômico de riqueza das espécies de hidroides bentônicos da CAAS e OA;

2) Reunir informações sobre seus substratos biológicos, endemismo, distribuições geográfica e batimétrica;

3) Investigar a (dis)similaridade entre as assembleias de hidroides bentônicos para a CAAS e OA;

4) Inferir áreas de endemismo para a CAAS e OA usando os hidroides bentônicos como modelo e a Análise de Endemicidade (AE) aplicada pelo software NDM-VNDM.

5) Reanalisar os dados de Marques \& Peña Cantero (2010 por meio da Análise de Parcimônia de Endemicidade (PAE) aplicada com registros complementares da distribuição de 61 espécies endêmicas de hidroides bentônicos para a região acima de $45^{\circ} \mathrm{S}$ dos oceanos Atlântico e Austral.

\section{OrganizaÇão Da TESE}

Esta tese é apresentada em cinco capítulos. Este capítulo faz uma introdução geral ao estudo, lista os principais objetivos da tese e descreve a forma como foi organizada. Os capítulos 2 a 4 são apresentados na forma de artigos científicos, um deles já publicado (capítulo 4), os quais possuem objetivos, resultados e discussões específicos e independentes, porém relacionados. O capítulo 5 apresenta as considerações finais e conclusões do estudo.

\section{REFERÊNCIAS}

Arntz, W.E. 1997. Investigación antártica en biología marina: situación actual, proyectos internacionales y perspectivas. Boletín de la Real Sociedad Española de Historia Natural, 93, 13-44.

Blanco, O. 1976. Hidrozoos de la expedición Walther Herwig. Revista del Museo de La Plata, 12, $27-74$. 
Blanco, O.M. 1994. Enumeración sistemática y distribución geográfica preliminar de Hydroida de la República Argentina: suborden Athecata (Gymnoblastea, Anthomedusae), Thecata (Calyptoblastea, Leptomedusae) y Limnomedusae. Revista del Museo de La Plata, 14, 181-216.

Bouillon, J., Gravili, C., Pagès, F., Gili, J.-M. \& Boero, F. 2006. An introduction to Hydrozoa. Mémoires du Muséum National d'Histoire Naturelle, 194, 1-591.

Brandt, A. 1999. On the origin and evolution of Antarctic Peracarida (Crustacea, Malacostraca). Scientia Marina, 63, 261-274.

Brandt, A., Linse, K. \& Mühlenhardt-Siegel, U. 1999. Biogeography of Crustacea and Mollusca of the Subantarctic and Antarctic regions. Scientia Marina, 63, 383-389.

De Broyer, C. \& Rauschert, M. 1999. Faunal diversity of the benthic amphipods (Crustacea) of the Magellan region as compared to the Antarctic (preliminary results). Scientia Marina, 63, 281-293.

Cañete, J.I., Leighton, G.L. \& Aguilera, F.F. 1999. Polychaetes from Aysén Fjord, Chile: distribution, abundance and biogeographical comparison with the shallow soft-bottom polychaete fauna from Antarctica and the Magellan Province. Scientia Marina, 63, 243-252.

Cartwright, P. \& Collins, A.G. 2007. Class Hydrozoa. In: Daly, M., Brugler, M.R., Cartwright, P., Collins, A.G., Dawson, M.N., Fautin, D.G., France, S.C., McFadden, C.S., Opresko, D.M., Rodriguez, E., Romano, S.L. \& Stake, J.L. The phyllum Cnidaria: a review of phylogenetic patterns and diversity 300 years after Linnaeus. Zootaxa, 1668, 127-182.

Clarke, A. 2008. Antarctic marine benthic diversity: patterns and processes. Journal of Experimental Marine Biology and Ecology, 366, 48-55.

Clarke, A. \& Crame, J.A. 1992. The Southern Ocean benthic fauna and climate change: a historical perspective. Philosophical Transactions of the Royal Society of London Series B Biological Sciences, 338, 299-309.

Clarke, A. \& Johnston, N.M. 2003. Antarctic marine benthic diversity. In: Gibson, R.N. \& Atkinson, R.J.A. (Eds) Oceanography and Marine Biology: an Annual Review. Taylor \& Francis, 41, 468p.

Clarke, A., Aronson, R.B., Crame, J.A., Gili, J.M. \& Blake, D.B. 2004. Evolution and diversity of the benthic fauna of the Southern Ocean continental shelf. Antarctic Science, 16, 559-568.

Clarke, A., Barnes, D.K.A. \& Hodgson, D.A. 2005. How isolated is Antarctic? Trends in Ecology and Evolution, 20, 1-3.

Clayton, M.N. 1994. Evolution of the Antarctic marine benthic algal flora. Journal of Phycology, 30, 897-904.

Crisci, J.V., Katinas, L. \& Posadas, P. 2000. Introducción a la teoría y práctica de la biogeografía histórica. Sociedad Argentina de Botânica, Buenos Aires, 169p.

Cunha, A.F. \& Jacobucci, G.B. 2010. Seasonal variation of epiphytic hydroids (Cnidaria: Hydrozoa) associated to a subtropical Sargassum cymosum (Phaeophyta: Fucales) bed. Zoologia, 27, 945955.

Galea, H.R. 2006a. On two new species of Halopteris Allman, 1877 (Cnidaria, Hydrozoa) from Chile. Zootaxa, 1165, 57-68.

Galea, H.R. 2006b. Rediscovery and redescription of Hybocodon chilensis Hartlaub, 1905 (Cnidaria, Hydrozoa) from Comau Fiord, southern Chile. Zootaxa, 1258, 57-68.

Galea, H.R. 2007. Hydroids and hydromedusae (Cnidaria: Hydrozoa) from the fjords region of southern Chile. Zootaxa, 1597, 1-116.

Galea, H.R. \& Schories, D. 2012. Some hydrozoans (Cnidaria) from Central Chile and the Strait of Magellan. Zootaxa, 3296, 19-67. 
Galea, H.R., G. Försterra \& V. Häussermann. 2007a. Additions to the hydroids (Cnidaria: Hydrozoa) from the fjords region of southern Chile. Zootaxa, 1650, 55-68.

Galea, H.R., Försterra, G. \& Häussermann, V. 2007b. Hydrozoa, fjord Comau, Chile. Check List, 3, 159-167.

Galea, H.R., Försterra, G. \& Häussermann, V. 2007c. Cnidaria, Hydrozoa: latitudinal distribution of hydroids along the fjords region of southern Chile, with notes on the world distribution of some species. Check List, 3, 308-320.

Galea, H.R., Försterra, G. \& Häussermann, V. 2009a. New additions to the hydroids (Cnidaria: Hydrozoa) from the fjords regions of southern Chile. Zootaxa, 2019, 1-28

Galea, H., Häussermann, V. \& Försterra, G. 2009b. Hydrozoa. In: Häussermann, V. \& Försterra, G. (Eds) Marine benthic fauna of Chilean Patagonia. Nature in Focus, 1000p.

Galea, H.R., Schories, D., Försterra, G. \& Häussermann, V. 2014. New species and new records of hydroids (Cnidaria: Hydrozoa) from Chile. Zootaxa, 3852, 1-50.

Genzano, G.N. 1998. Hydroid epizoites on hydroids Tubularia crocea and Sertularella mediterranea from the intertidal of Mar del Plata (Argentina). Russian Journal of Marine Biology, 24, 123126.

Genzano, G.N. 2001. Associated fauna and sediment trapped by colonies of Tubularia crocea (Cnidaria, Hydrozoa) from the rocky intertidal of Mar del Plata, Argentina. Biociências, 9, 105-119.

Genzano, G.N. 2002. Associations between pycnogonids and hydroids from the Buenos Aires litoral zone, with observations on the semi-parasitic life cycle of Tanystylum orbiculare (Ammotheiidae). Scientia Marina, 66, 83-92.

Genzano, G.N. \& Rodriguez, G.M. 1998. Association between hydroid species and their substrates from the intertidal zone of Mar del Plata (Argentine). Miscellània Zoològica, 21, 21-29.

Genzano, G.N. \& San Martín, G. 2002. Association between the polychaete Procerastea halleziana (Polychaeta: Syllidae: Autolytinae) and the hydroid Tubularia crocea (Cnidaria: Hydrozoa) from the Mar del Plata intertidal zone, Argentina. Cahiers the Biologie Marine, 43, 165-170.

Genzano, G.N. \& Zamponi, M.O. 1997. Frecuencia de estudio y diversidad de los hidrozoos bentónicos de la plataforma continental argentina. Ciencias Marinas, 23, 285-302.

Genzano, G.N. \& Zamponi, M.O. 2003. Hydroid assemblages from Mar del Plata, Argentina, at depths between 0 and $500 \mathrm{~m}$. Distribution and biological substrata. Oceanologica Acta, 25, 303-313.

Genzano ,G.N., Excoffon, A.C., Acuña, F.H. \& Zamponi, M.O. 2003. Hydroid colonies as primary substrata for recruits of the mussel Mytilus edulis platensis front off Mar del Plata, Argentina. Ophelia, 57, 53-61.

Genzano, G.N., Giberto, D., Schejter, L., Bremec, C. \& Meretta, P. 2009. Hydroid assemblages from the Souhwestern Atlantic Ocean (34-42 $\left.{ }^{\circ} \mathrm{S}\right)$. Marine Ecology, 30, 33-46.

Gerdes, D. \& Montiel, A. 1999. Distribution patterns of macrozoobenthos: a comparison between the Magellan region and the Weddell Sea (Antarctica). Scientia Marina, 63, 149-154.

Gili, J.-M. \& Hughes, R.G. 1995. The ecology of marine benthic hydroids. Oceanography and Marine Biology, an annual review, 33, 351-426.

Grohmann, P.A., Nogueira, C.C. \& Silva, V.M.A. 2003. Hydroids (Cnidaria, Hydrozoa) collected on the continental shelf of Brazil during the Geomar X Oceanographic Operation. Zootaxa, 299, 1-19.

Grohmann, P.A., Nogueira, C.C. \& Silva, V.M.A.P. 2011. Hydroids (Cnidaria, Hydrozoa) collected on the inner continental shelf of the state of Rio de Janeiro, Brazil, during the Oceanographic 
Operations GEOCOSTA RIO I and II. Biota Neotropica, 11, 193-201.

Hartlaub, C. 1905. Die Hydroiden der magalhaensishen region und chilenischen küste. Zoologische Jahrbücher, 6, 497-714.

Jäderholm, E. 1904. Hydroiden aus den Küsten von Chile. Arkiv för Zoologi, 2, 1-7.

Lawver, L.A. \& Gahagan, L.M. 2003. Evolution of Cenozoic seaways in the circum-Antarctic region. Palaeogeography, Palaeoclimatology, Palaeoecology, 198, 11-37.

Leloup, E. 1974. Hydropolypes calyptoblastiques du Chili. Report no. 48 of the Lund University Chile Expedition 1948-1949. Sarsia, 55, 1-62.

López-González, P.J. \& Gili, J.-M. 2000. A new octocoral genus (Cnidaria: Anthozoa) from Antarctic waters. Polar Biology, 23, 452-458.

Marques, A.C. \& Peña Cantero, A.L. 2010. Areas of endemism in the Antarctic - a case study of the benthic hydrozoan genus Oswaldella (Cnidaria, Kirchenpaueriidae). Journal of Biogeography, 37, 617-623.

Marques, A.C., Morandini, A.C. \& Migotto, A.E. 2003. Synopsis of knowledge on Cnidaria Medusozoa from Brazil. Biota Neotropica, 3, 1-18.

Marques, A.C., Klôh, A.S., Migotto, A.E., Cabral, A.C., Rigo, A.P.R., Bettim, A.L., Razzolini, E.L., Cascon, H.M., Bardi, J., Kremer, L.P., Vieira, L.M., Bezerra, L.E.A., Haddad, M.A., Oliveira Filho, R.R., Gutierre, S.M.M., Miranda, T.P., Franklin Jr., W. \& Rocha, R.M. 2013.

Rapid assessment survey for exotic benthic species in the São Sebastião Channel, Brazil. Latin American Journal of Aquatic Research, 41, 265-285.

Migotto, A.E. 1996. Benthic shallow-water hydroids (Cnidaria, Hydrozoa) of the coast of São Sebastião, Brazil, including a checklist of Brazilian hydroids. Zoologische Verhandelingen, 306, $1-125$.

Migotto, A.E. \& Marques, A.C. 2006. Invertebrados marinhos. In: Lewinsohn, T.M. (Ed.) Avaliação do estado do conhecimento da diversidade brasileira. Ministério do Meio Ambiente, 1, 149-202.

Migotto, A.E., Marques, A.C. \& Flynn, M.N. 2001. Seasonal recruitment of hydroids (Cnidaria) on experimental panels in the São Sebastião Channel, Southeastern Brazil. Bulletin of Marine Science, 68, 287-298.

Millard, N.A.H. 1975. Monograph on the Hydroida of Southern Africa. Annals of the South African Museum, 68, 1-513.

Miranda, T.P. 2009. Faunística e distribuição geográfica de hidróides bentônicos (Cnidaria, Hydrozoa) do sudoeste do Atlântico. Dissertação de mestrado. Instituto de Biociências, Universidade de São Paulo, 206p.

Miranda, T.P., Haddad, M.A., Shimabukuro, V., Dubiaski-Silva, J. \& Marques, A.C. 2011. Fauna de hidroides (Cnidaria, Hydrozoa) da região de Bombinhas, Santa Catarina, Brasil. Biota Neotropica, 11, 331-353.

Miranda, T.P., Peña Cantero, A.L. \& Marques, A.C. 2013. Southern Ocean areas of endemism: a reanalysis using benthic hydroids (Cnidaria, Hydrozoa). Latin American Journal of Aquatic Research, 41, 1003-1009.

Montiel, A.S.M., Gerdes, D. \& Arntz, W.E. 2005. Distributional patterns of shallow-water polychaetes in the Magellan region: a zoogeographical and ecological synopsis. Scientia Marina, $69,123-133$.

Morrone, J.J. 2001. Homology, biogeography and areas of endemism. Diversity and Distributions, 7, 297-300.

Mühlenhardt-Siegel, U. 1999. On the biogeography of Cumacea (Crustacea, Malacostraca). A 
comparison between South America, the Subantarctic Islands and Antarctica: present state of the art. Scientia Marina, 63, 295-302.

Oliveira, O.M.P. 2003. Diversidade e sazonalidade de hidróides (Cnidaria, Hydrozoa) epifíticos do Canal de São Sebastião, SP. Dissertação de Mestrado. Instituto de Biociências, Universidade de São Paulo, 109p.

Oliveira, O.M.P. \& Marques, A.C. 2007. Epiphytic hydroids (Hydrozoa: Anthoathecata and Leptothecata) of the world. Check List, 3, 21-38.

Oliveira, O.M.P., Marques, A.C. \& Migotto, A.E. 2006. Chave de identificação dos hidróides (Cnidaria, Hydrozoa) epifíticos do Canal de São Sebastião (SE, Brasil). Biota Neotropica, 6, $1-18$.

Oliveira, O.M.P., Araújo, E.M., Ayón, P., Cedeño-Posso, C.M., Cepeda, A.A., Córdova, P., Cunha, A.F., Galea, H., Genzano, G.N., Haddad, M.A., Mianzan, H.W., Migotto, A.E., Miranda, L.S., Miranda, T.P., Morandini, A.C., Nagata, R.M., Nascimento, K., Nogueira Jr., M., Palma, S., Quiñones, J.A.D., Rodriguez, C., Scarabino, F., Schiariti, A., Tronolone, V. \& Marques, A.C. Census of the Cnidaria (Medusozoa) and Ctenophora from South American marine waters. Zootaxa [in press].

Pagès, F. \& Orejas, C. 1999. Medusae, siphonophores and ctenophores of the Magellan region. Scientia Marina, 63, 51-57.

Peña Cantero, A.L. 2004. How rich is the deep-sea Antarctic benthic hydroid fauna? Polar Biology, $27,767-774$.

Peña Cantero, A.L. \& García Carrascosa, A.M. 1999. Biogeographical distribution of the benthic thecate hydroids collected during the Spanish "Antártida 8611" expedition and comparison between Antarctic and Magellan benthic hydroid faunas. Scientia Marina, 63, 209-218.

Rodriguez, C.S., Miranda, T.P., Marques, A.C., Mianzan, H. \& Genzano, G. 2012. The genus Hybocodon (Cnidaria, Hydrozoa) in the southwestern Atlantic Ocean, with a revision of the species recorded from the area. Zootaxa, 3523, 39-48.

Rosso, S. \& Marques, A.C. 1997. Patterns in intertidal hydrozoan distribution along the coast of São Paulo State, Southeastern Brazil. In: den Hartog, J.C. (Ed.) Proceedings of the 6th International Conference on Coelenterate Biology. National Natuurhistorisch Museum, 1st edition, 415-422.

Saiz-Salinas, J.I. \& Pagola-Carte, S. 1999. Sipuncula of the Magellan area compared with adjacent regions of Antarctica. Scientia Marina, 63, 227-232.

Schuchert, P. 1998. How many hydrozoan species are there? Zoologische Verhandelingen, 323, 209-219.

Schuchert, P. 2000. Hydrozoa (Cnidaria) of Iceland collected by the BIOICE programme. Sarsia, $85,411-438$.

Schuchert, P. 2001. Hydroids of the Greenland and Iceland (Cnidaria, Hydrozoa). Meddelelser om Grønland, Bioscience, 53, 1-184.

Shimabukuro, V. 2007. As associações epizóicas de Hydrozoa (Cnidaria: Leptothecata, Anthoathecata e Limnomedusae): I) Estudo faunístico de hidrozoários epizóicos e seus organismos associados, II) Dinâmica de comunidades bentônicas em substratos artificiais. Dissertação de Mestrado. Instituto de Biociências, Universidade de São Paulo, 275p.

Vannucci Mendes, M. Hydroida Thecaphora do Brasil. 1946. Arquivos de Zoologia do Estado de São Paulo, 4, 535-598.

Vannucci, M. 1949. Hydrozoa do Brasil. Boletim da Faculdade de Filosofia, Ciências e Letras da Universidade de São Paulo, 99, 219-266. 
Vannucci, M. 1951. Distribuição dos Hydrozoa até agora conhecidos nas costas do Brasil. Boletim do Instituto Paulista de Oceanografia, 2, 105-124. 


\section{Capítulo 5. Considerações finais}

A costa austral da América do Sul (CAAS) e o oceano Austral (OA) possuem enorme diversidade de habitats com diferentes características abióticas (Boltovskoy, 1981; Silva \& Palma, 2006; Silva, 2008; Genzano et al., 2009; Waller et al., 2011; Chown, 2012; Grange \& Smith, 2013). Ambas as áreas eram historicamente conectadas até que a passagem de Drake abriu-se há ca. 30 milhões de anos, separando a península antártica e a América do Sul (Barker \& Burrell, 1977; Beu et al., 1997; Lawver \& Gahagan, 2003; Clarke et al., 2005). Essa separação criou um sistema oceanográfico de correntes que isolou térmica e biogeograficamente o continente antártico, proporcionando o desenvolvimento de uma fauna altamente endêmica na região (Barker \& Burrell, 1977; Clarke \& Crame, 1989; Beu et al., 1997; Lawver \& Gahagan, 2003; Barker \& Thomas, 2004; Clarke et al., 2004).

Similaridades e correspondências entre as faunas marinhas de CAAS e OA têm sido melhor documentadas (e.g., Cañete et al., 1999; Peña Cantero \& García Carrascosa, 1999; Clarke \& Johnston, 2003; Clarke et al., 2005; Yasuhara et al., 2007; Marques \& Peña Cantero, 2010; Kaiser et al., 2011; Miranda et al., 2013), tornando essa região geográfica um modelo interessante com relação aos fatores que influenciaram sua história evolutiva (Clarke et al., 2004). A alta diversidade bentônica de CAAS e OA (Clarke \& Crame, 1992; De Broyer \& Rauschert, 1999; Peña Cantero \& García Carrascosa, 1999; Clarke et al., 2004; Giberto et al., 2004; Peña Cantero, 2004; Gappa et al., 2006; Brandt et al., 2007; Galea et al., 2007; Schejter \& Bremec, 2007; Yasuhara et al., 2007; Clarke, 2008; Genzano et al., 2011; Kaiser et al., 2013; Marques et al., 2013; Oliveira et al., in press), associada à sua história geológica e oceanográfica e a alta diversidade de habitats, a torna uma modelo para o desenvolvimento de estudos básicos em biogeografia, tais como a delimitação de áreas de endemismo.

Neste estudo, os hidroides bentônicos (exceto hidrocorais), um grupo com boa representatividade e sucesso de sobrevivência no bentos marinho, foi usado para estudar os padrões de endemismo da CAAS e OA. No total, 359 morfoespécies de hidroides bentônicos foram levantadas para a área de estudo, sendo 3 delas (?Nemertesia ciliata, Sertularella leiocarpa, Zygophylax sibogae) novos registros para o sudoeste do Atlântico. Dessas espécies, 256 foram identificadas até o nível específico, 40 até gênero, 44 têm o status taxonômico duvidoso e 19 não puderam ser identificadas devido a sua condição morfológica precária. Das 256 espécies, 54\% são endêmicas para a CAAS e SO, sendo a maioria Leptothecata (92\%).

A maior riqueza de espécies foi registrada para o sudoeste do Atlântico (73\% do total de espécies). Com relação à distribuição horizontal e vertical, as espécies estão relativamente bem distribuídas ao longo da CAAS e SO, sendo a maior parte das espécies encontradas até $200 \mathrm{~m}$ de profundidade. Exceções são as espécies de "Anthoathecata", as quais concentraram-se mais nas áreas tropicais e as espécies de Sertulariidae, Kirchenpaueriidae, Schizotrichidae, Lafoeidae e Plumulariidae, que foram mais frequentes em zonas mais profundas $(>200 \mathrm{~m})$. Com relação ao substrato, os hidroides bentônicos caracterizaram-se pela predominância de espécies generalistas, sendo a maior parte encontrada em substratos naturais.

As análises de similaridade de fauna por "clusters" e nMDS resultaram em 13 assembleias de hidroides bentônicos, sendo clara a separação entre a CAAS e OA. A Análise de Endemicidade (AE) feita pelo software NDM-VNDM encontrou $9(\mathrm{~F}=0,5)$ e $10(\mathrm{~F}=1,0)$ áreas consensuais de endemismo, com 107 e 112 espécies endêmicas no total, respectivamente. Os padrões de endemismo incluem composições únicas de hidroides bentônicos, concentrando-se principalmente em oito regiões: (1) sudoeste do Atlântico, (2) América do Sul, (3) América do Sul e Península 
Antártica, (4) Patagônia e Península Antártica, (5) Arco de Scotia e Península Antártica, (6) região Magalhânica e Península Antártica, (7) região Magalhânica e (8) Península Antártica e Mar de Ross. Esses padrões de endemismo podem ser explicados tanto por processos dispersivos quanto vicariantes. Algumas "áreas de endemismo" tiveram seu conceito de endemicidade questionado e discutido em relação a aspectos de biogeografia ecológica.

As assembleias e as áreas de endemismo de CAAS e OA resultantes tiveram seus padrões comparados a aspectos oceanográficos e outros padrões biogeográficos (ecológicos e históricos) previamente delimitados para a região (Spalding et al., 2007; Marques \& Peña Cantero, 2010; Miranda et al., 2013; Miranda et al., não publicado). Por outro lado, a Análise de Parcimônia de Endemicidade (PAE) foi realizadas com 61 hidroides bentônicos endêmicos da região acima dos $45^{\circ} \mathrm{S}$ dos oceanos Atlântico e Austral. Essa análise resultou em oito áreas de endemismo para a região subantártica e antártica, corroborando padrões gerais previamente encontrados por Marques \& Peña Cantero (2010), mas definindo as subregiões com maior grau de acurácia (viz., Miranda et al., 2013).

O conhecimento sobre a diversidade e biogeografia de hidroides bentônicos reunidos e trabalhados neste estudo permite que mudanças na biodiversidade possam ser melhor avaliadas, em especial face aos constantes e crescentes impactos ambientais acumulados no ambiente marinho. Os padrões biogeográficos ecológicos e históricos que descrevemos são uma tentativa de entender melhor o ambiente marinho e os fatores ambientais responsáveis por sua manutenção e evolução. Espera-se que a ampla base de dados aqui reunida colabore para elucidar outros padrões biogeográficos e macroecológicos de comunidades marinhas bentônicas, integrando diferentes abordagens biogeográficas e contribuindo para criação de estratégias e programas futuros em conservação da biodiversidade marinha.

\section{REFERÊNCIAS}

Barker, P.F. \& Burrell, J. 1977. The opening of Drake Passage. Marine Geology, 25, 15-34.

Barker, P.F. \& Thomas, E. 2004. Origin, signature and palaeoclimatic influence of the Antarctic Circumpolar Current. Earth-Science Reviews, 66, 143-162.

Beu, A.G., Griffin, M. \& Maxwell, P.A. 1997. Opening of Drake Passage gateway and Late Miocene to Pleistocene cooling reflected in Southern Ocean molluscan dispersal: evidence from New Zealand to Argentina. Tecnophysics, 281, 83-97.

Boltovskoy, D. 1981. Atlas del zooplankton del Atlántico Sudoccidental y métodos de trabajo con el zooplankton marino. Publicación Especial del INIDEP, 936p.

Brandt, A., De Broyer, C., De Mesel, I., Ellingsen, K.E., Gooday, A.J., Hilbig, B., Linse, K., Thomson, M.R.A. \& Tyler, P.A. 2007. The biodiversity of the deep Southern Ocean benthos. Philosophical Transactions of the Royal Society, 362, 39-66.

De Broyer, C. \& Rauschert, M. 1999. Faunal diversity of the benthic amphipods (Crustacea) of the Magellan region as compared to the Antarctic (preliminary results). Scientia Marina, 63, 281-293.

Cañete, J.I., Leighton, G.L. \& Aguilera, F.F. 1999. Polychaetes from Aysén Fjord, Chile: distribution, abundance and biogeographical comparison with the shallow soft-bottom polychaete fauna from Antarctica and the Magellan Province. Scientia Marina, 63, 243-252.

Chown, S.L. 2012. Antarctic marine biodiversity and deep-sea hydrothermal vents. PLoS Biology, 10, e1001232.

Clarke, A. 2008. Antarctic marine benthic diversity: patterns and processes. Journal of Experimental Marine Biology and Ecology, 366, 48-55. 
Clarke, A. \& Crame, J.A. 1989. The origin of the Southern Ocean marine fauna. In: Crame, J.A. (Ed.) Origins and evolution of the Antarctic biota. Geological Society Special Publication, 47, $322 \mathrm{p}$.

Clarke, A. \& Crame, J.A. 1992. The Southern Ocean benthic fauna and climate change: a historical perspective. Philosophical Transactions of the Royal Society of London Series B Biological Sciences, 338, 299-309.

Clarke, A. \& Johnston, N.M. 2003. Antarctic marine benthic diversity. In: Gibson, R.N. \& Atkinson, R.J.A. (Eds) Oceanography and Marine Biology: an Annual Review. Taylor \& Francis, 41, 468p.

Clarke, A., Aronson, R.B., Crame, J.A., Gili, J.-M. \& Blake, D.B. 2004. Evolution and diversity of the benthic fauna of the Southern Ocean continental shelf. Antarctic Science, 16, 559-568.

Clarke, A., Barnes, D.K.A. \& Hodgson, D.A. 2005. How isolated is Antarctica? Trends in Ecology and Evolution, 20, 1-3.

Galea, H.R., Försterra, G. \& Häussermann, V. 2007. Cnidaria, Hydrozoa: latitudinal distribution of hydroids along the fjords region of southern Chile, with notes on the world distribution of some species. Check List, 3, 308-320.

Gappa, J.L., Alonso, G.M. \& Landoni, N.A. 2006. Biodiversity of benthic Amphipoda (Crustacea: Peracarida) in the Southwest Atlantic between $35^{\circ} \mathrm{S}$ and $56^{\circ} \mathrm{S}$. Zootaxa, 1342, 1-66.

Genzano, G.N., Giberto, D., Schejter, L., Bremec, C. \& Meretta, P. 2009. Hydroid assemblages from the Souhwestern Atlantic Ocean (34-42 $\left.{ }^{\circ} \mathrm{S}\right)$. Marine Ecology, 30, 33-46.

Genzano, G., Giberto, D. \& Bremec, C. 2011. Benthic survey of natural and artificial reefs off Mar del Plata, Argentina, southwestern Atlantic. Latin American Journal of Aquatic Research, 39, 553-566.

Giberto, D.A., Bremec, C.S., Acha, E.M. \& Mianzan, H. 2004. Large-scale spatial patterns of benthic assemblages in the SW Atlantic: the Río de La Plata estuary and adjacent shelf waters. Estuarine Coastal and Shelf Science, 61, 1-13.

Grange, L.J. \& Smith, C.R. 2013. Magafaunal communities in rapidly warming fjords along the West Antarctic Peninsula: hotspots of abundance and beta diversity. PLoS ONE, 8, e77917.

Kaiser, S., Griffiths, H.J., Barnes, D.K.A., Brandão, S.N., Brandt, A. \& O’Brien, P.E. 2011. Is there a distinct continental slope fauna in the Antarctic? Deep-Sea Research II, 58, 91-104.

Kaiser, S., Brandão, S.N., Brix, S., Branes, D.K.A., Bowden, D.A., Ingels, J., Leese, F., Schiaparelli, S., Arango, C.P., Badhe, R., Bax, N., Blazewicz-Paszkowycz, M., Brandt, A., Brenke, N., Catarino, A.I., David, B., De Ridder, C., Dubois, P., Ellingsen, K.E., Glover, A.G., Griffiths, H.J., Gutt, J., Halanych, K.M., Havermans, C., Held, C., Janussen, D., Lörz, A.-N., Pearce, D.A., Pierrat, B., Riehl, T., Rose, A., Sands, C.J., Soler-Membrives, A., Schüller, M., Strugnell, J.M., Vanheusel, A., Veit-Köhler, G., Wilson, N.G. \& Yasuhara, M. 2013. Patterns, processes and vulnerability of Southern Ocean benthos: a decadal leap in knowledge and understanding. Marine Biology, 160, 2295-2317.

Lawver, L.A. \& Gahagan, L.M. 2003. Evolution of Cenozoic seaways in the circum-Antarctic region. Palaeogeography, Palaeoclimatology, Palaeoecology, 198, 11-37.

Marques, A.C. \& Peña Cantero, A.L. 2010. Areas of endemism in the Antarctic - a case study of the benthic hydrozoan genus Oswaldella (Cnidaria, Kirchenpaueriidae). Journal of Biogeography, $37,617-623$.

Marques, A.C., Klôh, A.S., Migotto, A.E., Cabral, A.C., Rigo, A.P.R., Bettim, A.L., Razzolini, E.L., Cascon, H.M., Bardi, J., Kremer, L.P., Vieira, L.M., Bezerra, L.E.A., Haddad, M.A., Oliveira Filho, R.R., Gutierre, S.M.M., Miranda, T.P., Franklin Jr., W. \& Rocha, R.M. 2013. 
Rapid assessment survey for exotic benthic species in the São Sebastião Channel, Brazil. Latin American Journal of Aquatic Research, 41, 265-285.

Miranda, T.P., Peña Cantero, A.L. \& Marques, A.C. 2013. Southern Ocean areas of endemism: a reanalysis using benthic hydroids (Cnidaria, Hydrozoa). Latin American Journal of Aquatic Research, 41, 1003-1009.

Miranda, T.P., Genzano, G.N. \& Marques, A.C. Endemicity in benthic hydroids (Cnidaria, Hydrozoa) in the Southwestern Atlantic ocean: an NDM-VNDM hypothesis [não publicado].

Oliveira, O.M.P., Araújo, E.M., Ayón, P., Cedeño-Posso, C.M., Cepeda, A.A., Córdova, P., Cunha, A.F., Galea, H., Genzano, G.N., Haddad, M.A., Mianzan, H.W., Migotto, A.E., Miranda, L.S., Miranda, T.P., Morandini, A.C., Nagata, R.M., Nascimento, K., Nogueira Jr., M., Palma, S., Quiñones, J.A.D., Rodriguez, C., Scarabino, F., Schiariti, A., Tronolone, V. \& Marques, A.C. Census of the Cnidaria (Medusozoa) and Ctenophora from South American marine waters. Zootaxa [in press].

Peña Cantero, A.L. 2004. How rich is the deep-sea Antarctic benthic hydroid fauna? Polar Biology, 27, 767-774.

Peña Cantero, A.L. \& García Carrascosa, A.M. 1999. Biogeographical distribution of the benthic thecate hydroids collected during the Spanish "Antártida 8611" expedition and comparison between Antarctic and Magellan benthic hydroid faunas. Scientia Marina, 63, 209-218.

Schejter, L. \& Bremec, C. 2007. Benthic richness in the Argentine continental shelf: the role of Zygochlamys patagonica (Mollusca: Bivalvia: Pectinidae) as settlement substrate. Journal of Marine Biological Association of the United Kingdom, 87, 917-925.

Silva, N. 2008. Dissolved oxygen, $\mathrm{pH}$, and nutrients in the austral Chilean channels and fjords. In: Silva, N. \& Palma, S. (Eds) Progress in the oceanographic knowledge of Chilean interior waters, from Puerto Montt to Cape Horn. Comité Oceanográfico Nacional, Pontificia Universidad Católica de Valparaíso, 161p.

Silva, N. \& Palma, S. 2006. E1 Programa CIMAR en los canals y fiordos australes. In: Silva, N. \& Palma, S. (Eds) Avances en el conocimiento oceanográfico de las aguas interiors chilenas, Puerto Montt a cabo de Hornos. Comité Oceanográfico Nacional, Pontificia Universidad Católica de Valparaíso, 161p.

Spalding, M.D., Fox, H., Allen, G.R., Davidson, N., Ferdaña, Z.A., Finlayson, M., Halpern, B.S., Jorge, M.A., Lombana, A., Lourie, S.A., Martin, K.D., McManus, E., Molnar, J., Recchia, C.A. \& Robertson, J. 2007. Marine ecoregions of the world: a bioregionalization of coastal and shelf areas. BioScience, 57, 573-583.

Waller, R.G., Scanlon, K.M. \& Robinson, L.F. 2011. Cold-water coral distributions in the Drake Passage Area from towed camera observations - initial interpretations. PLoS ONE, 6, e16153.

Yasuhara, M., Kato, M., Ikeya, N. \& Seto, K. 2007. Modern benthic ostracodes from Lützow-Holm Bay, East Antarctica: paleoceanographic, paleobiogeographic, and evolutionary significance. Micropaleontology, 53, 469-496. 